12. Hopke, J., Donath, J., Blechert, S. \& Boland, W. Herbivore-induced volatiles: the emission of acyclic homoterpenes from leaves of Phaseolus lunatus and Zea mays can be triggered by a $\beta$-glucosidase and jasmonic acid. FEBS Lett. 352, 146-150 (1994).

13. Drukker, B., Scutareanu, P. \& Sabelis, M. W. Do anthocorid predators respond to synomones from Psylla-infested pear trees under field conditions? Entomol. Exp. Appl. 77, 193-203 (1995).

14. Shimoda, T., Takabayashi, J., Ashihara, W. \& Takafuji, A. Response of predatory insect Scolothrip takahashii toward herbivore-induced plant volatiles under laboratory and field conditions. J. Chem. Ecol. 23, 2033-2048 (1997).

15. De Moraes, C. M., Lewis, W. J., Paré, P. W., Alborn, H. T. \& Tumlinson, J. H. Herbivore-infested plant selectively attract parasitoids. Nature 393, 570-572 (1998).

16. Boland, W., Hopke, J., Donath, J., Nuske, J. \& Bublitz, F. Jasmonic acid and coronatin induce odor production in plants. Angew. Chem. Int. Ed. Engl. 34, 1600-1602 (1995).

17. Alborn, H. T. et al. An elicitor of plant volatiles from beet armyworm oral secretion. Science 276, 945 949 (1997)

18. Campbell, B. C. \& Duffey, S. S. Tomatine and parasitic wasps: Potential incompatibility of plantantibiosis with biological control. Science 205, 700-702 (1979).

19. Strand, L. L. Integrated Pest Management for Tomatoes (Division of Agriculture and Natural Resource Communication Services-Publications, Oakland, 1998).

20. Takabayashi, J. \& Dicke, M. Plant-carnivore mutualism through herbivore-induced carnivore attractants. Trends Plant Sci. 1, 109-113 (1996).

21. Clancy, K. M. \& Price, P. W. Rapid herbivore growth enhances enemy attack: sublethal plant defenses remain a paradox. Ecology 68, 733-737 (1987).

22. Benrey, B. \& Denno, R. F. The slow-growth-high-mortality hypotheses: a test using the cabbage butterfly. Ecology 78, 987-999 (1997)

23. Stout, M. J., Workman, K. V. \& Duffey, S. S. Identity, spatial distribution, and variability of induced chemical responses in tomato plants. Entomol. Exp. Appl. 79, 255-271 (1996).

24. Bell, E. \& Mullet, J. E. Characterization of an Arabidopsis lipoxygenase gene responsive to methyl jasmonate and wounding. Plant. Physiol. 103, 1133-1137 (1993).

25. Siedow, J. N. Plant lipoxygenase: structure and function. Annu. Rev. Plant Physiol. Plant Mol. Biol. 42, 145-188 (1991).

26. Paré, P. W., Alborn, H. T. \& Tumlinson, J. H. Concerted biosynthesis of an insect elicitor of plant volatiles. Proc. Natl Acad. Sci. USA 95, 13971-13975 (1998).

27. Dicke, M., Sabelis, M. W., Takabayashi, J., Bruin, J. \& Posthumus, M. A. Plant strategies of manipulating predator-prey interactions through allelochemicals: prospects for application to pest control. J. Chem. Ecol. 16, 3091-3118 (1990).

Acknowledgements. I thank C. Wardlaw and C. Black for help in the field; M. Stout and C. De Moraes for discussion; and A. Agrawal, R. Karban, M. Dicke, J. Rosenheim, L. Adler, C. Black, R. Kimura and $\mathrm{J}$. Granett for comments on the manuscript. This research was supported by the USDA-NRI.

Correspondence and requests for materials should be addressed to J.S.T. (e-mail: jsthaler@ucdavis.edu)

\section{Auditory cortical responses in the cat to sounds that produce spatial illusions}

\section{Li Xu, Shigeto Furukawa \& John C. Middlebrooks}

Kresge Hearing Research Institute, University of Michigan, 1301 E. Ann St, Ann Arbor, Michigan 48109-0506, USA

Humans and cats can localize a sound source accurately if its spectrum is fairly broad and flat $^{1-3}$, as is typical of most natural sounds. However, if sounds are filtered to reduce the width of the spectrum, they result in illusions of sources that are very different from the actual locations, particularly in the up/down and front/ back dimensions ${ }^{4-6}$. Such illusions reveal that the auditory system relies on specific characteristics of sound spectra to obtain cues for localization ${ }^{7}$. In the auditory cortex of cats, temporal firing patterns of neurons can signal the locations of broad-band sounds $s^{8-9}$. Here we show that such spike patterns systematically mislocalize sounds that have been passed through a narrow-band filter. Both correct and incorrect locations signalled by neurons can be predicted quantitatively by a model of spectral processing that also predicts correct and incorrect localization judgements by human listeners ${ }^{6}$. Similar cortical mechanisms, if present in humans, could underlie human auditory spatial perception.

Resonance in the cavities of the external ears, combined with diffraction by the head, act to enhance particular frequencies in an arriving sound and to cancel other frequencies. The filter properties of the head and ears vary according to the direction of incidence of a sound, so details of the frequency spectra at the eardrums can reveal the direction of the source. Interpretation of spectral cues for localization is confounded when sound sources are filtered experimentally ${ }^{2-6}$. For instance, in a study in which stimuli were restricted to $1 / 6$-octave frequency bands, human listeners reported a compel- ling illusion of a sound source that deviated in vertical or front/back location from the actual source ${ }^{6}$. Regardless of the stimulus location, a typical listener in that study consistently reported hearing sound sources high and in front when the centre frequency of the stimulus spectrum was $6 \mathrm{kHz}$, low and generally to the front when the centre frequency was $8 \mathrm{kHz}$, and low and generally to the rear when the centre frequency was $10 \mathrm{kHz}$. An acoustical model based on measurements of the filter characteristics of each listener's ears successfully predicted the listener's locations judgments. Listeners appeared to interpret narrow-band stimulus spectra as if they were broad-band spectra that had been filtered by their own external ears. In cats, two recent studies ${ }^{2-3}$ have demonstrated localization behaviour that was generally consistent with human results. Like humans, cats localized broad-band sounds accurately ${ }^{2-3}$ and made large localization errors when presented with 1/6-octave narrowband sounds ${ }^{3}$. Unlike humans, the cats' responses did not vary systematically with stimulus centre frequency. That negative result, however, might reflect the response measure that was used in the cat experiments, which constrained the cats' responses to a limited frontal area. Our localization model predicts that the narrow-band stimuli that were tested in that study would have produced illusory targets high above or behind the cats.

In this study, we recorded unit activity from cortical area A2 in cats that were anaesthetized with $\alpha$-chloralose ${ }^{8}$. Area A2 was chosen because neurons in that area respond to broad ranges of frequency and because elevation sensitivity tends to be greater there than in the adjacent anterior ectosylvian sulcus area ${ }^{9}$ or in area A1 (unpublished observations). We used silicon-substrate thin-film probes that permitted simultaneous recording of unit activity at up to 16 cortical sites ${ }^{10}$. Custom spike-sorting software was used to isolate single units or small clusters of units. Each sound was presented in an anechoic room from one of 14 loudspeakers. The loudspeakers were located in the vertical midline, all at a distance of $1.2 \mathrm{~m}$ from the centre of the cat's head. We use the term elevation to refer to a continuous angular dimension measured relative to the frontal horizontal plane and extending up and over the head to the rear. Stimulus locations ranged in elevation in $20^{\circ}$ increments from $-60^{\circ}$ (below the horizontal plane in front), through $+90^{\circ}$ (straight overhead) to $+200^{\circ}\left(20^{\circ}\right.$ below the horizontal plane in the rear). Stimuli consisted of filtered gaussian noise bursts, $80 \mathrm{~ms}$ long. Sound pressures were varied in 5- or $10-\mathrm{dB}$ steps between 20 and $40 \mathrm{~dB}$ above neural thresholds. Broad-band filter spectra were flat and extended from 1 to $30 \mathrm{kHz}$. Narrow-band filters had 1/6-octavewide flat centres, skirts that fell off at $120 \mathrm{~dB}$ per octave and centre frequencies that ranged from 4 to $18 \mathrm{kHz}$. We recorded from 389 single units and small clusters of units in area A2 of eight cats. When tested with tonal stimuli, most of the units responded to frequencies in the upper half of the cat's audible range, and all showed frequency bandwidths of an octave or more at levels $40 \mathrm{~dB}$ above threshold.

Neurons typically responded to broad-band noise bursts with one or a few spikes locked to the onset of the stimulus. Spike probabilities and spike latencies varied according to the elevation of the sound source. As in previous studies ${ }^{8,9}$, we computed estimates of spike density functions (spike patterns) by forming averages of multiple samples of eight responses to each stimulus; samples were drawn with replacement. Spike patterns drawn from odd-numbered trials were used to train an artificial neural network; we then used the trained network to estimate sound-source elevations by classifying spike patterns drawn from even-numbered trials. Figure 1a shows the network estimates of source elevations based on the spike patterns of one cortical neuron. In this example of broad-band stimulus conditions, responses generally clustered around the dashed line that represented perfect performance.

The accuracy of elevation estimates under broad-band conditions, as represented by the medians of unsigned errors, ranged from $19.9^{\circ}$ to around the chance-performance level of $65^{\circ}$. Responses to narrow-band stimuli were analysed for the half of 


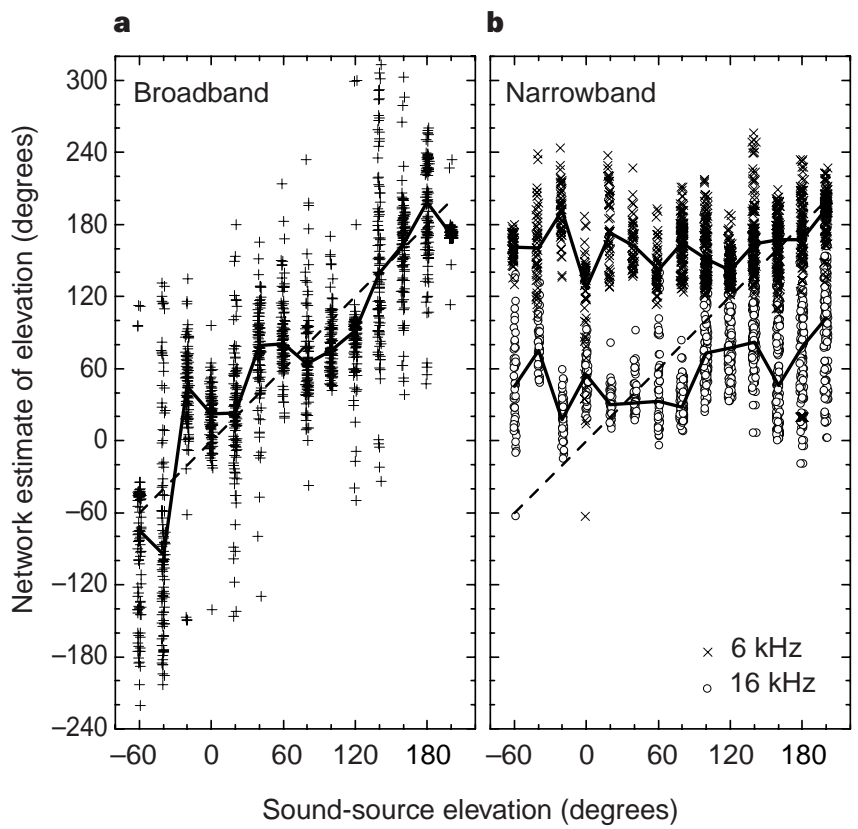

Figure 1 Artificial-neural-network analysis of spike patterns from a single unit (9806C16). The network was trained to estimate stimulus elevations based on spike patterns elicited by broad-band sounds that varied in 5-dB steps from 20 to $40 \mathrm{~dB}$ above neural threshold. The trained network was used to classify spike patterns elicited by broad-band (a) or narrow-band (b) sounds. Each symbol represents the network estimate of sound-source elevation based on an average of eight responses from one unit. The samples of eight responses were drawn with replacement. The dashed line indicates perfect correspondence between stimulus location and the network estimate. The solid line shows the median

the unit sample (194 units, median errors $\leq 50.4^{\circ}$ ) that showed the most accurate elevation coding; complete data for narrow-band centre frequencies between 6 and $18 \mathrm{kHz}$ were obtained for 112 to 146 units, depending on the frequency. For each unit, we trained an artificial neural network with spike patterns from broad-band conditions, then used the trained network to classify spike patterns that were elicited by 1/6-octave band-passed stimuli at various centre frequencies. We regard this procedure as a physiological analogue of requiring a psychophysical listener to report the apparent location of a narrow-band sound. Neuronal elevation sensitivity was markedly reduced when narrow-band stimuli were used. Figure $1 \mathrm{~b}$ shows the network estimates of source elevations based on neuronal responses to noise bands centred at 6 and $16 \mathrm{kHz}$. Like the responses of human listeners in narrow-band localization tasks, unit responses were largely insensitive to source elevation and tended to vary according to the centre frequencies of the stimulus spectra. In this example, the network localized the $6-\mathrm{kHz}$ stimulus as up and to the rear and the $16-\mathrm{kHz}$ stimulus as up and in front. In this $16-\mathrm{kHz}$ example, unit responses to front targets differed somewhat from those to rear targets, but elevation estimates were far from accurate.

To implement a model that related sound localization to externalear acoustics ${ }^{6}$, we measured the filter characteristics of the external ears of the cats that were studied physiologically. The filter characteristics were represented by directional transfer functions (DTFs $)^{9,11}$. DTFs were measured by recording from the ear canals with microphones as a broad-band sound source was varied systematically in location. DTFs were similar among cats, in that upward elevation shifts of the sound source generally resulted in upward frequency shifts of spectral peaks and notches. Nevertheless, there was variability in the details of DTFs among cats that was at least qualitatively similar to the variability observed among human subjects $^{12}$. The acoustical model involved a computation of the value at each source location. a, Classification of responses to broad-band sounds. Stimulus levels were $20 \mathrm{~dB}$ above neural threshold. The median error across all source elevations was $31.5^{\circ}$. b. Classification of responses to $1 / 6$ octave noise bands centred at 6 and $16 \mathrm{kHz}$. Stimulus levels were $40 \mathrm{~dB}$ above threshold. Unit responses to narrow-band sounds varied with stimulus centre frequency but were largely insensitive to sound-source elevation. When artificial neural networks were trained and tested with responses to narrow-band sound, performance was consistently worse than for broad-band conditions, generally around chance levels.

differences between stimulus spectra and DTFs (Fig. 2a). For each of the narrow-band stimuli and the DTF measured at each elevation, we subtracted the amplitude spectrum (gain) of the DTF from the stimulus spectrum, then computed the variance of the resulting difference distribution. That difference metric is referred to here as the spectral difference. In our previous psychophysical work ${ }^{6}$, listeners' localization judgements tended to fall at elevations for which their DTFs were most similar to the stimulus spectrum. Similarly, we predicted that the responses of cortical neurons would signal elevations that minimized the spectral difference between the stimulus spectrum and the cats' DTFs. Figure $2 \mathrm{~b}$ and $\mathrm{c}$ shows the spectral differences computed for two cats for a range of stimulus centre frequencies. In these plots, each vertical column of coloured squares represents the spectral differences computed between a stimulus of a particular centre frequency and the DTFs at various elevations. The differences between the two panels are a result of differences between cats in the frequencies of components of their DTFs.

The plus signs in Fig. $2 \mathrm{~b}$ and c represent artificial-neural-network estimates of elevation based on the responses of one neuron to narrow-band stimuli at various centre frequencies. The plus signs tend to fall on or near the yellow squares that indicate small spectral differences, which would be the predicted responses in a psychophysical task. The differences in cortical responses between Fig. $2 \mathrm{~b}$ and $2 c$ presumably reflect differences in acoustical input that resulted from differences in DTFs.

We tested the correspondence between cortical responses and model predictions by ranking spectral differences at each stimulus centre frequency (each vertical column in plots like Fig. $2 b$ and c) for each cat. Low ranks indicate small spectral differences. On each physiological trial, we scored the rank of the elevation at which the network estimate fell. The median rank was recorded for each centre frequency for each of the units. Figure 3 shows the distribution of 


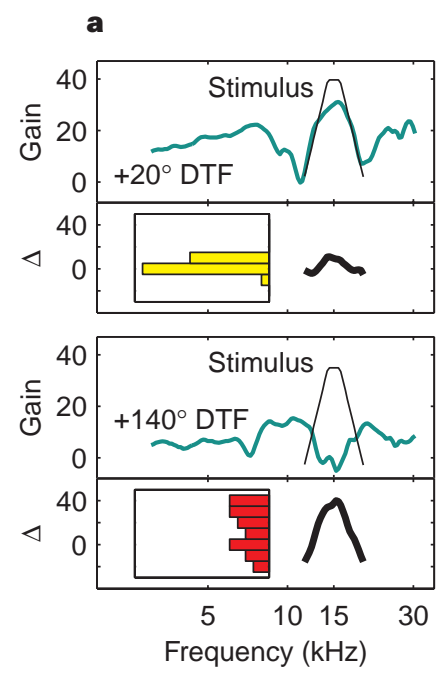

Figure 2 Spectral differences and network classification of cortical responses to narrow-band sounds. a, Spectral differences represent the differences between stimulus filter functions (thin black lines) and DTFs (green lines). The examples show a narrow-band stimulus centred at $15 \mathrm{kHz}$ and DTFs for elevations of +20 and $+140^{\circ}$. Filter functions and DTFs were expressed as gain in $\mathrm{dB}$. Spectra were subtracted, frequency component by frequency component, to obtain difference spectra $(\Delta$, thick black lines). The spectral difference metric was given by the variance of the resulting difference distribution (yellow and red histograms). b, Spectral differences and network classifications for unit 9806C16. Spectral

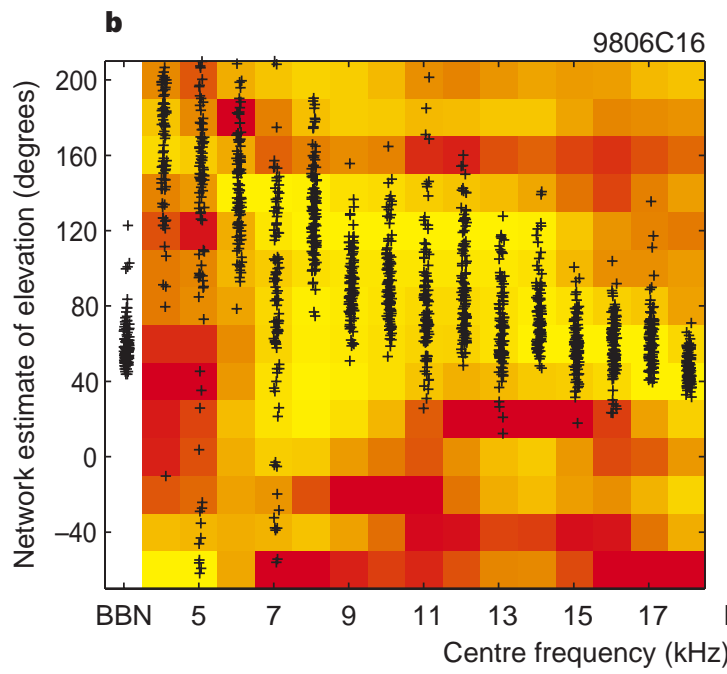

c
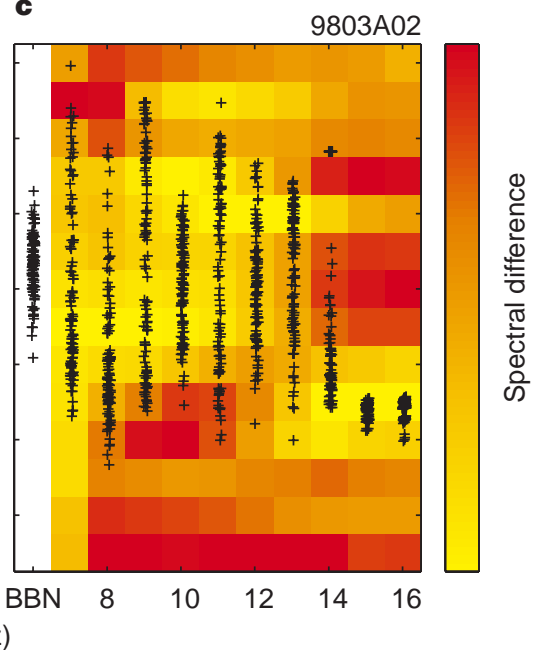

differences are represented by colours. Values at each stimulus centre frequency were scaled to span the full colour range. Light yellow indicates a small spectral difference, which indicates a prediction of a high likelihood of a response at the elevation. Symbols indicate network classifications of cortical responses to narrow-band stimuli. All sound sources were located at $+80^{\circ}$ elevation. The vertical column labelled BBN indicates the network classification of cortical responses to a broad-band sound presented from $+80^{\circ}$ elevation. c, Spectral variances and network classifications for unit 9803A02. Other conventions as in $\mathbf{b}$.

median ranks across all units. Low-numbered ranks indicate good model performance, and chance performance would produce ranks around 7.5. Model performance was quite good for most units for centre frequencies between 11 and $16 \mathrm{kHz}$. Performance approached chance levels at lower and higher frequencies. It has been shown that cats can localize in elevation fairly accurately when tested with noise-band stimuli that are restricted to $10-14 \mathrm{kHz}$, whereas performance is near chance when stimuli are restricted to lower or higher frequencies ${ }^{2}$.

Our results show successful prediction of cat neuronal signalling by a model that was derived to account for human psychophysical performance. Neurons signal accurately the elevations of broadband sounds that humans localize accurately. In addition, in

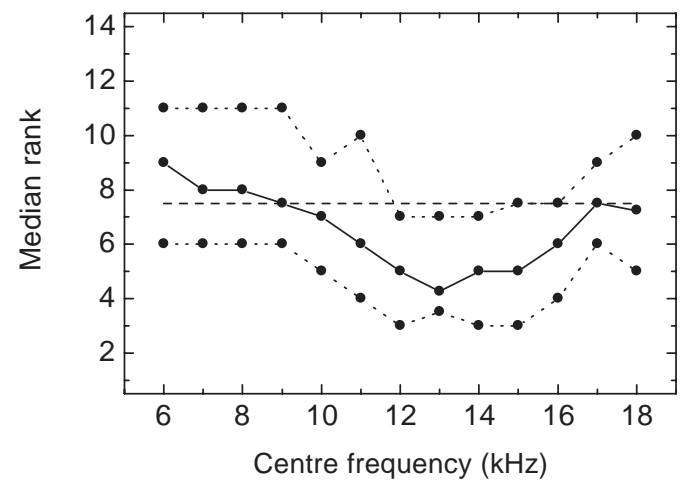

Figure 3 Summary of correspondence between physiological responses and the acoustical model. At each centre frequency, each unit was represented by a median rank as described in the text. Ranks less than 7.5 (dashed line) represent better-than-chance performance. A distribution of 112 to 146 median ranks was compiled at each centre frequency. The solid line represents the medians of the distributions and the dotted lines represent the $25^{\text {th }}$ and $75^{\text {th }}$ percentiles. Data shown are with stimulus levels fixed at $40 \mathrm{~dB}$ above neural thresholds. Similar results were obtained in 20- and $30-\mathrm{dB}$ fixed-level conditions and with levels varying in 10-dB steps between 20 and $40 \mathrm{~dB}$ above threshold.

response to narrow-band sounds, neurons signal incorrect locations that can be predicted by a model that predicts incorrect human localization. We would like to interpret this correspondence between psychophysical and physiological results as evidence that cortical responses analogous to those recorded in the cat could underlie human perception. That interpretation, of course, involves a conceptual jump that crosses the gap between species, cat to human, and crosses the gap across levels, from neurons to perception. Although procedural differences prevent an exact comparison between psychophysical results in humans and cats, particularly in regard to narrow-band stimuli, sound localization appears at least qualitatively similar between species. The jump across levels, from neurons to perception, is a general problem in neuroscience that cannot be solved by this study. Nevertheless, the results of this study do offer strong support for the conclusion that neuronal elevation sensitivity in cats and spatial hearing in humans obey similar computational principles. Neurons like those that we record in that cat auditory cortex, if present in humans, could play a key role in auditory spatial perception.

Received 16 March; accepted 21 April 1999.

1. Makous, J. C. \& Middlebrooks, J. C. Two-dimensional sound localization by human listeners. J. Acoust. Soc. Am. 87, 2188-2200 (1990).

2. Huang, A. Y. \& May, B. J. Sound orientation behavior in cats. II. Mid-frequency spectral cues for sound localization. J. Acoust. Soc. Am. 100, 1070-1080 (1996).

3. Populin, L. C. \& Yin, T. C. Behavioral studies of sound localization in cat. J. Neurosci. 18, 2147-2160 (1998).

4. Blauert, J. Sound localization in the median plane. Acustica 22, 205-213 (1969-1970).

5. Hebrank, J. \& Wright, D. Spectral cues used in the localization of sound sources on the median plane. J. Acoust. Soc. Am. 56, 1829-1834 (1974).

6. Middlebrooks, J. C. Narrow-band sound localization related to external ear acoustics. J. Acoust. Soc. Am. 92, 2607-2624 (1992).

7. Middlebrooks, J. C. \& Green, D. M. Sound localization by human listeners. Annu. Rev. Psychol. 42, 135-159 (1991).

8. Middlebrooks, J. C., Xu, L., Eddins, A. C. \& Green, D. M. Codes for sound-source location in nontonotopic auditory cortex. J. Neurophysiol. 80, 863-881 (1998).

9. Xu, L., Furukawa, S. \& Middlebrooks, J. C. Sensitivity to sound-source elevation in non-tonotopic auditory cortex. J. Neurophysiol. 80, 882-894 (1998).

10. Najafi, K., Wise, K. D. \& Mochizuki, T. A high-yield IC-compatible multichannel recording array. IEEE Trans. Electron Devices 32, 1206-1211 (1985).

11. Middlebrooks, J. C. \& Green, D. M. Directional dependence of interaural envelope delays. J. Acoust. Soc. Am. 87, 2149-2162 (1990).

12. Middlebrooks, J. C. Individual differences in external-ear transfer functions reduced by scaling in frequency. J. Acoust. Soc. Am. (in press). 
Acknowledgements. We thank Z Onsan for expert technical assistance, and E. Macpherson, B. Mickey, D. Moody, B. Pfingst and J. Schacht for comments on the manuscript. This work was supported by the NIH/HIHCD. The multi-channel recording probes were supplied by the Center for Neural Communication Technology, which is supported by the NIH/NCRR

\section{The SIL gene is required for mouse embryonic axial development and left-right specification}

Shai Izraeli*, Linda A. Lowe $\dagger$, Virginia L. Bertness*, Deborah J. Good‡, David W. Dorward\$, Ilan R. Kirsch* \& Michael R. Kuehn $\dagger$

* Genetics Department, Medicine Branch, National Cancer Institute, NIH, Bethesda, Maryland 20889-5105, USA

$\dagger$ Experimental Immunology Branch, National Cancer Institute, NIH, Bethesda, Maryland 20892-1360, USA

$\ddagger$ Department of Veterinary and Animal Sciences, University of Massachusetts, Amherst, Massachusetts 01003, USA

$\$$ Rocky Mountain Laboratories, National Institute of Allergy and Infectious Diseases, NIH, Hamilton, Montana 59840, USA

The establishment of the main body axis and the determination of left-right asymmetry are fundamental aspects of vertebrate embryonic development. A link between these processes has been revealed by the frequent finding of midline defects in humans with left-right anomalies ${ }^{1}$. This association is also seen in a number of mutations in mouse ${ }^{2-4}$ and zebrafish ${ }^{1,5}$, and in experimentally manipulated Xenopus embryos ${ }^{5}$. However, the severity of laterality defects accompanying abnormal midline development varies ${ }^{6}$, and the molecular basis for this variation is unknown. Here we show that mouse embryos lacking the earlyresponse gene $S I L$ have axial midline defects, a block in midline Sonic hedgehog (Shh) signalling and randomized cardiac looping. Comparison with $S h h$ mutant embryos ${ }^{7}$, which have axial defects but normal cardiac looping, indicates that the consequences of abnormal midline development for left-right patterning depend on the time of onset, duration and severity of disruption of the normal asymmetric patterns of expression of nodal, lefty-2 and Pitx2.

SIL is an early-response gene that was discovered at the site of a leukaemia-associated chromosomal rearrangement ${ }^{8}$. It is ubiquitously expressed in proliferating cells ${ }^{9}$ and during early embryonic development (not shown). To analyse SIL function in vivo, we deleted exons 3-5, including the translation start $\operatorname{sites}^{10}$, by homologous recombination in murine embryonic stem cells (not shown). Immunoblot analysis of protein extracts from $S I L^{-/-}$cells confirmed that this is a null allele (not shown). Mice derived from two independent $S I L^{-1-}$ embryonic stem-cell clones showed the same phenotype. Heterozygotes are normal, but mutant homozygotes die in utero after embryonic day 10.5 (E10.5). Between E7.5 and E8.5, striking developmental anomalies appear in $S I L^{-1-}$ embryos. In addition to reduced size and limited developmental progress compared to wild-type embryos (Fig. 1a, b), SIL mutants display prominent midline neural-tube defects. These include delay or failure of neural-tube closure and a holoprosencephalic defect, namely the lack of any midline separation at the anterior end of the cranial neural folds (Fig. 1c, d). In addition, left-right development is abnormal. In heterozygous and wild-type embryos the embryonic heart tube always loops to the right (Fig. 1e), whereas in SIL mutants the direction of heart looping is randomized (Fig. 1f, g; Table 1).

The transforming growth factor- $\beta$ (TGF- $\beta$ ) family members
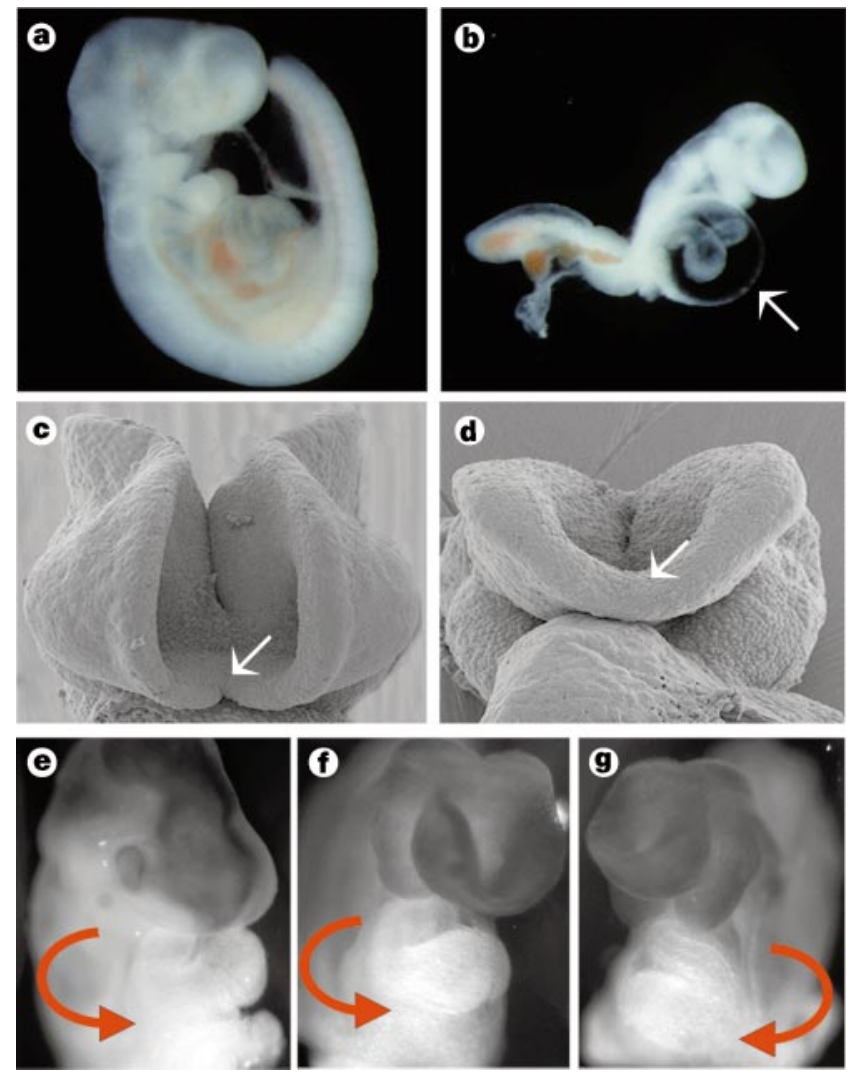

Figure 1 Gross morphology of $S / L^{-/-}$embryos. a, Lateral view of normal E9.5 embryo. b. E9.5 SIL mutant embryo, unturned with abnormal pericardial swelling (arrow). c, Scanning electron microscopy (SEM) of normal E8.5 embryo head, frontal view. Arrow, separation at anterior of neural folds. d, SEM of E8.5 SIL

embryo, lacking normal midline separation at the anterior of the neural folds (arrow). e, Ventral view of normal E9.5 embryo showing normal rightwards heart looping (direction shown by red arrow). f, E9.5SIL ${ }^{-/-}$embryo with rightwards heart looping. g, E9.5 S/L ${ }^{-/-}$embryo with leftwards heart looping.

nodal, lefty-1 and lefty-2 and the transcription factor Pitx2 are all implicated in the establishment of left-right-handed asymmetry in the mouse ${ }^{11-18}$. Nodal, lefty-2 and Pitx2 are normally expressed only in the left lateral-plate mesoderm (LPM) before heart looping, with continued expression of Pitx2 on the left side of the looping heart tube (Fig. $2 \mathrm{a}-\mathrm{c}$ ). In contrast, SIL mutants showed bilaterally symmetric expression of nodal and Pitx2 (Fig. 2d, f) at all stages examined (Table 1). For lefty-2, most $S I L^{-l-}$ embryos also showed bilaterally symmetric expression (Fig. 2e). However, a small number of mutants expressed lefty-2 only on the right. These were all at very early somite stages, when lefty-2 expression is first detectable (Table 1), indicating that abnormal lefty-2 expression on the right side of SIL mutants may precede normal left-sided expression. In normal embryos lefty- 1 is expressed on the left side of the prospective floorplate (PFP) at the ventral midline of the neural folds. In SIL mutants we found no midline lefty expression at any stage examined (Fig. 2e). These results show that SIL activity is required to establish the normal pattern of left-right asymmetric gene expression, both in the LPM and in the PFP, from the earliest stages of their expression.

The observed neural-tube defects, including the lack of lefty-1 expression in the PFP, prompted an analysis of midline developmental pathways. Two key players in midline development are the secreted signalling factor Shh and the winged helix transcription factor HNF-3 $\beta$ (refs 7, 19, 20). Both are normally expressed in the node and notochord at E7.5 to E8.5 (Fig. 3a, g for Shh; 3c, e, i, k for $H N F-3 \beta)$. HNF-3 $\beta$ is found also in the PFP (Fig. 3i, k), where its expression is dependent on Shh signalling from the adjacent node 